\section{Resultados del tratamiento del cáncer de mama, Programa Nacional de Cáncer del Adulto}

\author{
CÉSAR DEL CASTILLO SM. ${ }^{1}$, M. ELENA CABRERA C. ${ }^{2,3}$, \\ LEA DERIO P., ,a, FANCY GAETE V. ${ }^{5}$, GABRIEL CAVADA CH.,
}

\section{Impact of the Chilean Explicit Guaranties Health System (GES) on breast cancer treatment}

Background: Breast cancer is the most common malignant tumor in women in the world. In 2005, it was incorporated to the Explicit Guaranties Health System (GES) in Chile. Aim: To describe the demographic and clinical characteristics of breast cancer patients and to determine the effect of incorporating these women to GES. Material and Methods: Medical records of 5,119 women with breast cancer aged $59 \pm 14$ years, attended at six public hospitals between 2000 and 2010 were reviewed. Median follow up was 87 months (range 1-182). Mortality was assessed using death certificates obtained at the National Identification Registry. Results: Sixty six percent of women were in stage I-II, 29\% in stage III and 5\% in stage IV. Surgery was performed in 4023/5119 cases (79\%), adyuvant radiotherapy in $3627 / 4517$ cases (80\%), chemotherapy in 3,204/3,424 cases (94\%) and hormone therapy in 1,695/2,375 cases (71\%). Between 2000 and 2010, there was a significant increase in the proportion of cases in stage I, from $8 \%$ to $25 \%,(p<0.01)$. Overall survival (OS) increased $1 \%$ per year, since the beginning of GES system $(p=0.024)$. Five year OS was $75.1 \%$. The figures for Stage I, II, III and IV were 93, 84, 62 and 27\% respectively $(p<0.01) . P a-$ tients without lymph node involvement and who were not triple negative, had a significantly better OS. Conclusions: There was a significant increase in stage I cases, and a 1\% per year OS improvement after GES system started, compared with the previous period.

(Rev Med Chile 2017; 145: 1507-1513)

Key words: Breast Neoplasm; Chile; Therapeutics; Triple Negative Breast Neoplasm.

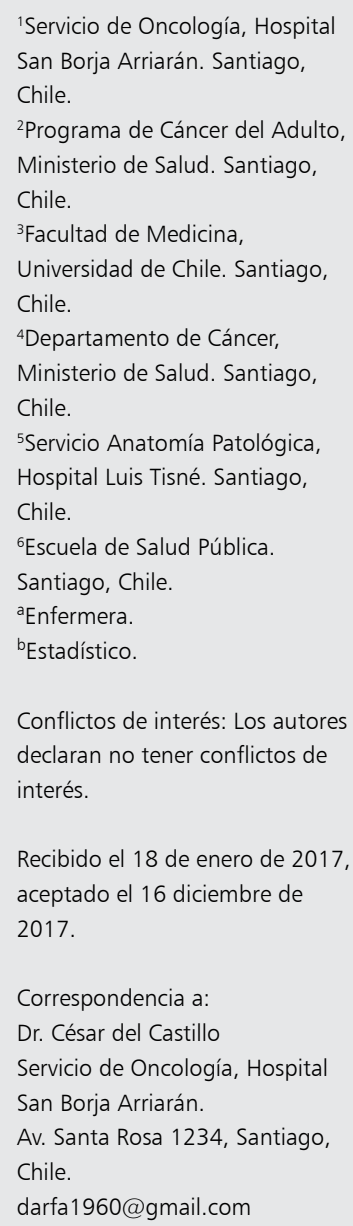

E 1 cáncer de mama constituye la primera causa de muerte en la mujer en Chile. En este sentido, el Programa de Cáncer del Adulto del Ministerio de Salud de Chile (PANDA), se ha preocupado desde la pesquisa precoz, el tratamiento (quirúrgico, quimioterapia, radioterapia, hormonoterapia y rehabilitación) hasta los cuidados paliativos.
La tasa estandarizada de incidencia a nivel mundial es de $39 \times 100.000$ mujeres/año y en Chile de 40.1 x 100.000 mujeres/año, siendo de 51.8 en mujeres de 35 a 64 años (Globocan 2008). Por su parte, la mortalidad ha descendido en muchos países europeos ${ }^{1}$. Esta declinación se ha atribuido a efecto combinado de mejor tamizaje (screening) y mejores tratamientos. En Chile, la tasa ajustada 
de mortalidad también muestra un descenso desde $14,12 / 100.000$ mujeres en el año 2000, a 12,63 en el año 2012 (DEIS, Minsal).

El cáncer de mama se incorporó al sistema de Garantías Explícitas en Salud (GES) en Chile, que garantiza a toda la población susceptible a acceso, oportunidad, cobertura financiera y calidad de la atención integral, en el mes de julio de 2005. El objetivo de este estudio fue describir las características demográficas y clínicas de los pacientes con diagnóstico de cáncer de mama registrados y tratados en los centros de cáncer de la red pública de salud de la Región Metropolitana (RM), entre los años 2000 y 2010, la sobrevida global (SG), así como determinar si se ha producido un cambio en la presentación clínica de esta neoplasia hacia etapas más precoces, desde el ingreso de esta patología al GES.

\section{Material y Método}

Se obtuvo la información desde las fichas clínicas de los pacientes tratados en los seis centros públicos de cáncer del adulto de la RM, entre los años 2000 y 2010 , transcritas a una base de datos resguardada en el Departamento de Cáncer del Ministerio de Salud. Se mantuvo la confidencialidad de la información. Se consideró pertenecientes a pueblos originarios aquellos pacientes que tenían al menos un apellido autóctono. El diagnóstico de cáncer de mama, se realizó por biopsia. Desde el año 2008, se consideraron los marcadores hormonales por inmunohistoquímica (IHQ), receptores de estrógeno y progesterona. La determinación del receptor humano de crecimiento epidérmico (Human-Epidermal-Growth-factor-receptor 2, HER2) se inició en abril de 2011, por lo que no fue evaluado en este estudio. Los pacientes fueron estadificados con hemograma, bioquímica general, radiografía de tórax, ecografía abdominal y cintigrama óseo. Se definió el estadío clínico inicial según el sistema $\mathrm{TNM}^{1}$. La mamografía fue incorporada al sistema público en el año 2005.

Desde el inicio del estudio estaba financiada la cirugía, radioterapia (RT), quimioterapia (QT) para todos los estadíos y la hormonoterapia (tamoxifeno). En el año 2004, se incorporaron los taxanos y los inhibidores de aromatasa. Los tratamientos se presentan en la Tabla $1^{2}$. En suma, en los estadíos I, II y III resecables se realizó cirugía: mastectomía parcial (MP), con disección axilar (DA) o mastectomía total extendida (MT), RT, QT y hormonoterapia adyuvante. En los estadios III irresecables, QT neoadyuvante ${ }^{3}$. Todos los casos fueron evaluados en Comité Oncológico del establecimiento, para decidir la conducta terapéutica y los pacientes firmaron consentimiento informado para su tratamiento.

La sobrevida global (SG) se midió desde la fecha de biopsia diagnóstica inicial hasta la fecha de último control o muerte. No se evaluó la sobrevida libre de recaída. La fecha y causa de muerte se obtuvo de certificados de defunción emitidos por el Servicio de Registro Civil e Identificación. Se evaluó la SG a 5 años, según estadío clínico y triple negativo, la que se describió según KaplanMeier y comparada mediante el test de Log-rank. Las asociaciones de la sobrevida con las variables continuas se analizaron mediante el modelo de riesgo proporcional de Cox, se construyó con la misma metodología el modelo multivariante para evaluar los factores pronósticos en conjunto. Las variables continuas fueron descritas mediante promedio y desviación estándar y las variables categóricas mediante frecuencias y proporciones.

Tabla 1. Tratamiento PANDA cáncer de mama según etapa clínica

\begin{tabular}{|llll|}
\hline Etapa & Cirugía & Quimioterapia & Hormonoterapia \\
\hline I y II & MP + DA + RT & 6 FAC o 4 AC o 6 CMF & Tamoxifeno y/o inh. aromatasa \\
III resecable & MT + RT & 6 FAC & Tamoxifeno y/o inh. aromatasa \\
III irresecable & QT + MT + RT & 6 FAC & \\
IV & QT & 6 FAC + Taxanos & Tamoxifeno y/o inh. aromatasa \\
\hline
\end{tabular}

$\mathrm{MP}=$ mastectomía parcial, $\mathrm{MT}=$ mastectomía total, $\mathrm{DA}=$ disección axilar, $\mathrm{RT}=$ radioterapia, $\mathrm{QT}=$ quimioterapia, inh. = inhibidores aromatasa. 
Los datos fueron procesados es STATA (versión 12.0). Los intervalos de confianza son de nivel $95 \%$ y se utilizó una significación de 5\%.

\section{Resultados}

Se analizaron 5.119 registros de pacientes con diagnóstico de cáncer de mama, de los siguientes establecimientos: Instituto Nacional del Cáncer 1.600 casos $(31,3 \%)$, Hospital de Salvador/Hospital Luis Tisné 1.430 casos (27,9\%), Hospital Sótero del Río 1.103 casos (21,5\%), Hospital San Juan de Dios 390 casos (7,6\%), Hospital Barros Luco Trudeau 353 casos $(6,9 \%)$ y Hospital Clínico San Borja Arriarán 243 casos (4,7\%). Ciento ocho pacientes se consideraron de pueblos originarios $(2,1 \%)$, de ellos, 103 mapuche y 5 pascuense. Cuatro pacientes fueron de sexo masculino $(0,08 \%)$. La distribución por edad al diagnóstico se muestra en la Figura 1. El promedio de edad fue 58,6 años $\pm 13,6$ (rango 18-102), < 35 años $2,7 \%$ (138 casos), $<50$ años $29,8 \%$ (1.526 casos) y > 50 años $67,5 \%$ (3.455 casos).

El número de linfonodos comprometidos fue registrado en 4.473 casos $(87,4 \%)$ y su distribución fue: 0 linfonodo: 49,9\% (2.234 casos); 1-3 linfonodos, 27,0\% (1.206 casos); 4-9 linfonodos, $14,7 \%$ (659 casos) y $\geq 10$ linfonodos, $8,4 \%$ (374 casos).

En 4.693 casos $(91,7 \%)$ se registró el estadio clínico, distribuyéndose en estadio I: 18,0\% (846 casos), estadio II: $47,7 \%$ (2.238 casos), estadio III: $29,1 \%$ (1.368 casos) y estadio IV: 5,1\% (241 casos). Es decir, $65,7 \%$ se presentaron en estadios localizados (I y II). La diseminación metastásica fue solo ósea en 103 casos, asociada a compromiso pulmonar en 29 y compromiso hepático en 24, pulmonar exclusiva en 75 casos y asociada a compromiso hepático en 11, compromiso hepático exclusivo en 13 casos y cerebral en un caso.

El subtipo histológico fue consignado en 4.960 casos $(96,9 \%)$ y la histología más común fue el carcinoma ductal infiltrante, con 79,3\% (3.932 casos), luego, carcinoma lobulillar infiltrante: 10,2\% (504 casos), carcinoma ductal in situ: 5,2\% ( 260 casos), carcinoma lobulillar in situ: $0,4 \%$ (22 casos), ambos ductal y lobulillar: 0,4\% (22 casos), ductal mucinoso: $1,8 \%$ (87 casos), ductal medular: $1,0 \%$ (51 casos), inflamatorio: $0,1 \%$ (5 casos) y otros tumores: $1,6 \%$ (77 casos).

El estudio de marcadores hormonales estrogénicos fue positivo en 2.290/3.740 casos estudiados $(61,2 \%)$ y progesterona fue positivo en $1.192 / 3.053$ casos estudiados (39\%). Sesenta y siete de 637 casos con estudio completo de marcadores hormonales fueron catalogados como "triple negativo" $(10,5 \%)$.

\section{Tratamiento}

La cirugía se realizó en 4.023 pacientes (78,6\%): MT en 2.048 casos, con disección axilar en 1.271 y MP en 1.975 casos, con DA en 1.842 . Se realizó estudio de linfonodo centinela en 72 casos $\mathrm{y}$ fue positivo en 30 .

Se realizó RT adyuvante en 3.627/4.517 pacientes $(80,3 \%)$ en los que estuvo disponible la información. La información de la QT recibida estuvo disponible en $3.204 / 3.424$ casos $(93,6 \%)$

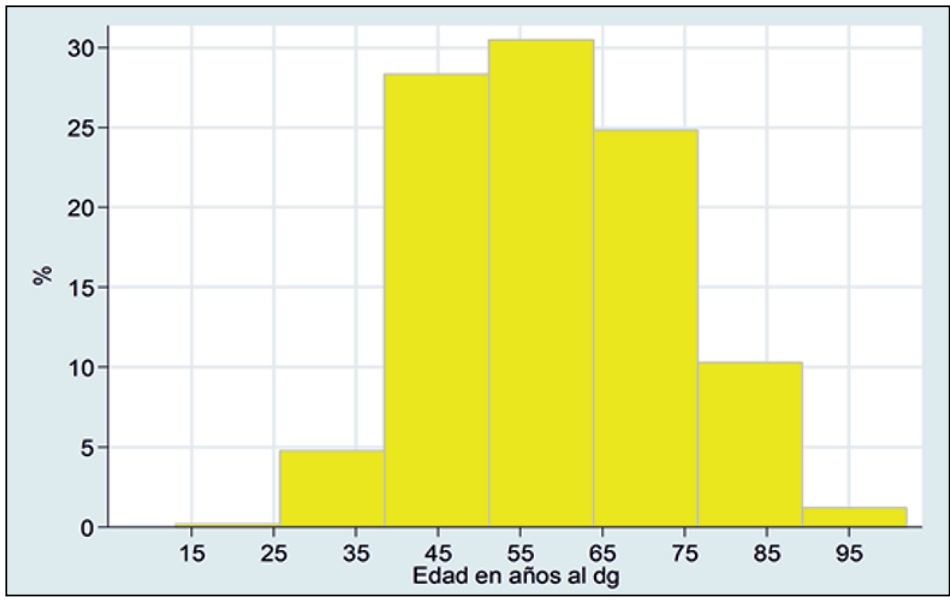

Figura 1. Distribución de la edad al diagnóstico de 5.119 casos de cáncer de mama. 
y fue adyuvante en 1.364 casos $(58,1 \%)$ y neoadyuvante en 984 casos (41,9\%). El esquema de QT dependió del período de tratamiento: 6 ciclos ciclofosfamida+metotrexato+fluoruracilo (CMF) en 93 casos, 4 ciclos adriamicina+ciclofosfamida (AC) +4 ciclos CMF en 273 casos, 4 ciclos $\mathrm{AC}$ en 1.801 casos, 6 ciclos fluoruracilo+adriamicinatciclofosfamida (FAC) en 815 casos, $\mathrm{AC}+$ taxanos en 179 casos, taxanos en 26 casos y FAC + CMF en 17 casos. La información de la hormonoterapia estuvo disponible en 1.695/2.375 casos (46,4\%): tamoxifeno en 1.593 casos, inhibidores de aromatasa en 100 y ambos, en dos. La ooforectomía se realizó en 145 casos.

\section{Sobrevida}

El seguimiento medio fue 87 meses (rango 1-182). La causa de muerte fue conocida en $1.743 / 2.037$ casos $(85,5 \%)$ : cáncer de mama en 1.434 casos $(82,2 \%)$ y otras neoplasias en 104 (6\%), destacando las del tubo digestivo: cáncer gástrico 18 , colon 8 , recto 6 , vesícula biliar 9 y páncreas 9. El otro grupo de neoplasias fue hematológica: leucemia mieloide aguda/síndrome mielodisplástico secundario a QT en 12 casos, linfoma no Hodgkin 2 casos y un caso de leucemia mieloide crónica. Las causas no neoplásicas fueron de origen infeccioso en 86 casos y cardiovascular en 68 casos.

La SG a 5 años del grupo completo fue 75,1\%. La SG por estadio fue: estadio I: 93,3\%, estadio II:
84,2\%, estadio III: $61.8 \%$ y estadio IV: $26,6 \%$ (Figura 2), presentado una gradiente epidemiológica significativa $(p=0,001)$. La edad no fue un factor pronóstico adverso $(p=0,07)$. La SG a 5 años de acuerdo al número de linfonodos axilares comprometidos en 4.473 casos con el dato conocido, fue $84,5 \%, 80,6 \%, 68,2 \%$ y $54,9 \%$, con $0,1-3,4-9$ $\mathrm{o} \geq 10$ ganglios positivos, respectivamente ( $\mathrm{Fi}-$ gura 3$)$, diferencia estadísticamente significativa $(\mathrm{p}=0,0001)$.

La SG a 5 años entre los dos subtipos histológicos más comunes, carcinoma ductal y lobulillar infiltrante, fue similar: $75,5 \%$ y $74,9 \%$, respectivamente $(p=0,72)$. En cambio, los cinco casos con carcinoma inflamatorio presentaron una sobrevida significativamente menor (33\%) a 5 años.

El grupo triple negativo presentó una SG a 5 años significativamente inferior en comparación con los casos no triple negativo, 64,0 y 79,6\%, respectivamente $(\mathrm{p}=0,002)$ (Figura 4$)$.

\section{Impacto del GES}

Entre los años 2000 y 2010, se observó un aumento significativo de los casos diagnosticados en estadio I, de $8,4 \%$ a $24,7 \%$, respectivamente $(\mathrm{p}<0,001)$ (Figura 5). También se evaluó la SG en este período. Entre 2000 y 2005, la SG a 5 años se mantuvo sin variación ( $\mathrm{p}=0,139)$, en cambio en el período post GES, 2005-2010, se observó una tendencia significativa al alza $(\mathrm{p}=0,005)$, de $1 \%$ por año $(\mathrm{p}=0,024)$ (Figura 6$)$.

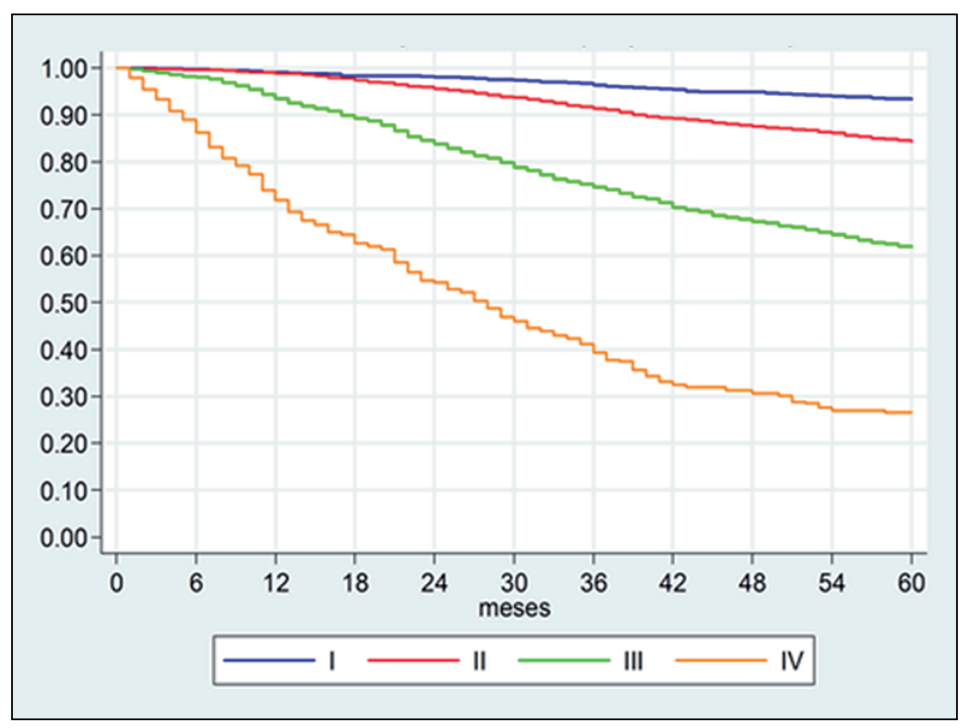

Figura 2. Sobrevida de acuerdo al estadio clínico al diagnóstico de 4.793 casos de cáncer de mama. 

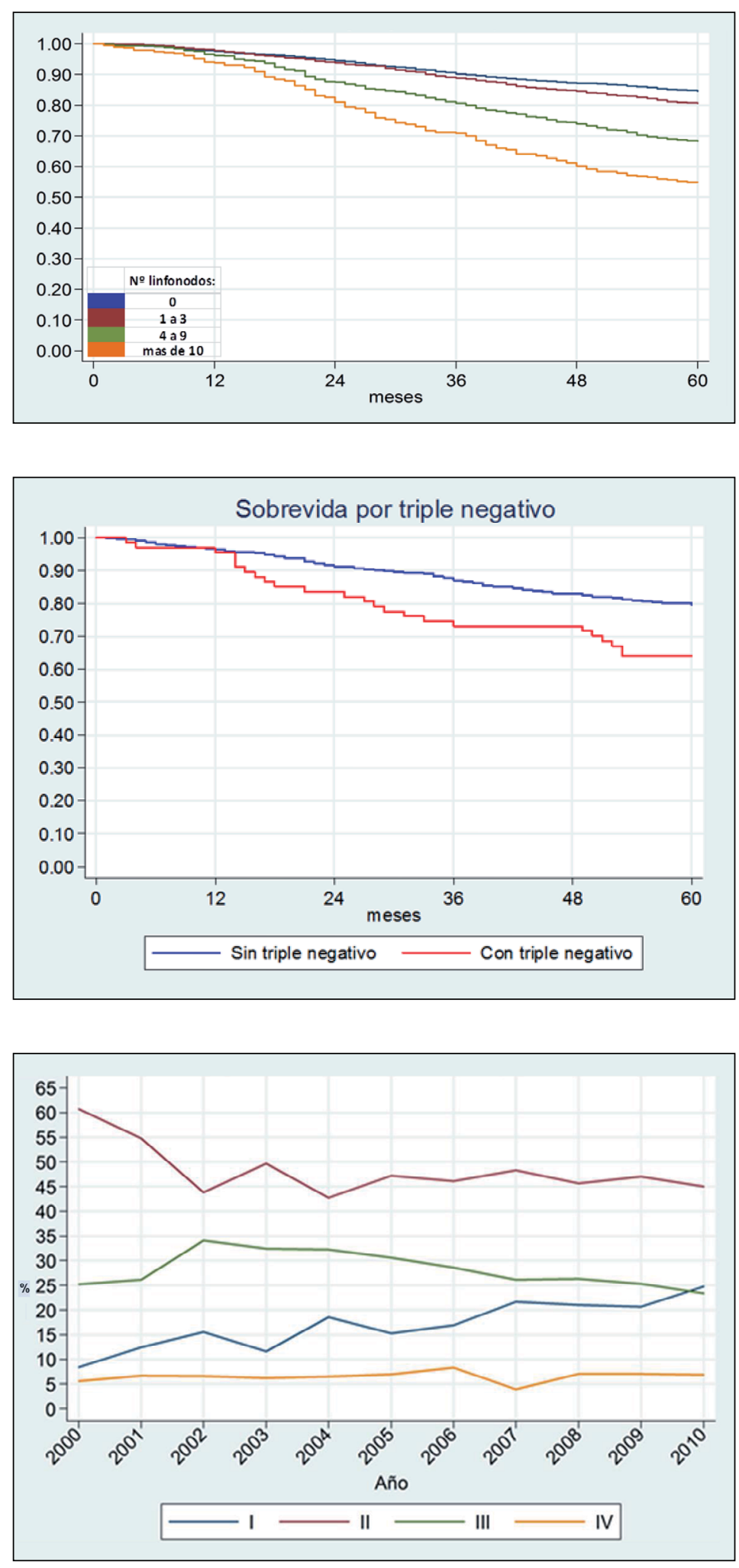

Figura 3. Sobrevida de acuerdo al número de linfonodos comprometidos en 4.443 casos de cáncer de mama.
Figura 4. Sobrevida global de 67 pacientes con cáncer de mama triple negativo, comparado con 637 casos no triple negativo.
Figura 5. Estadio clínico al diagnóstico de cáncer de mama, de acuerdo al año de diagnóstico $(n=5.119)$. 


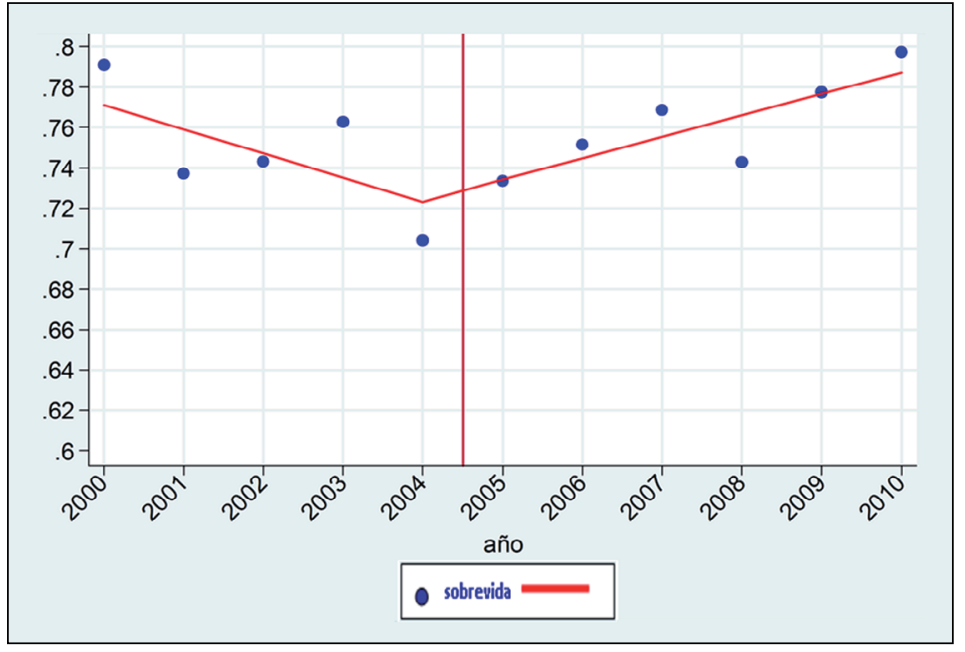

Figura 6. Sobrevida de acuerdo a fecha de diagnóstico pre y post GES, en cáncer de mama $(n=5.119)$.

\section{Discusión}

La presente cohorte muestra que en el período 2000-2010 se observó un aumento significativo del número de casos de cáncer de mama diagnosticados en estadio I a partir del inicio del programa GES y, también, una mejoría de la SG de todos los casos de cáncer de mama, de 1\% anual en el período post GES, respecto al período pre GES.

La explicación de esta tendencia, semejante a lo que se observa en países desarrollados, podría atribuirse a las políticas públicas del Ministerio de Salud, consistentes en un programa integral de acuerdo a la historia natural de este tumor y las variables relacionadas con las características de las mujeres chilenas. Si se observan los registros poblacionales de cáncer CONCORD $-2^{4}$ entre 2005-09 y se compara la SG de mujeres con cáncer de mama de diversos países con Chile, se puede apreciar que la SG a 5 años de Chile fue $75,1 \%$, inferior a Estados Unidos de Norteamérica (USA) y Europa, que tienen sobrevida superiores a $80 \%$, como USA 88,6\%, Reino Unido 81,1\%, España $83,7 \%$ o Suecia $86,2 \%$. Sin embargo, es similar a otros países latinoamericanos como Argentina $76,6 \%$, Colombia $76 \%$ o Cuba $77,9 \%$ o países de Europa del este, como Polonia $74,1 \%$ y superior a países como Malasia $68 \%$ o Sudáfrica $53 \%{ }^{4}$.

La tasa ajustada de mortalidad por cáncer de mama a su vez, ha disminuido en 1,5 puntos en 10 años, desde el inicio del GES en Chile (2005-2015), sin experimentar el descenso ocurrido en el Reino Unido y USA, de 20 y 10 puntos, respectivamente, luego de una estrategia sostenida y coherente en estos países por más de 20 años (1970-1990). Esperamos que este descenso ocurra en los próximos 10 años en nuestro país.

El aumento significativo del número de casos diagnosticados en estadio I en el período de estudio pone de manifiesto la utilidad de la detección precoz 5 . La sobrevida de $93,3 \%$ de este importante número de pacientes, 846 casos, es similar al estudio publicado de 125 mujeres en etapas precoces, T1N0M0 con cáncer de mama, tratadas en el Hospital Clínico de la Universidad Católica con cirugía conservadora $+\mathrm{RT}$, que describe una sobrevida de $96,5 \%{ }^{6}$.

Respecto al pequeño grupo de pacientes $(5,1 \%)$ que se presentaron con cáncer metastásico, estadio IV, la SG a 5 años de 26,6\% fue inferior a la publicada por el grupo de Sánchez y $\operatorname{col}^{7}$ de $39,8 \%$. Sin embargo, el seguimiento medio de esta cohorte fue solo de 26,7 meses, en comparación con 87 meses de nuestro grupo.

Respecto a la frecuencia de casos triple negativo, $10,5 \%$, fue similar a otro estudio chileno ${ }^{8}$ de $9,5 \%$ y al rango inferior observado en la literatura, 10-20\%, y también asociado a un pronóstico significativamente inferior. Aún no hay un tratamiento claro que mejore el pronóstico de estas pacientes ${ }^{9}$.

El presente estudio muestra el mayor número de casos con diagnóstico de cáncer de mama publi- 
cado en Chile. El objetivo del Programa Nacional de Cáncer es mejorar las medidas de detección y realizar diagnóstico en etapas más precoces, con educación sobre el autoexamen, mamografía de screening y realizar tratamientos adecuados, oportunos y de calidad, para mejorar la sobrevida de las mujeres con cáncer de mama.

Se propone ampliar los rangos de edad de la mamografía y aumentar la periodicidad a 2 años, tal como recomienda la Estrategia Nacional de Salud de Chile 2011-2020 ${ }^{10}$.

Agradecimientos: Dr. Rodrigo Arriagada por los comentarios al manuscrito. Dr. Juan Pablo Miranda Olivares por los comentarios al manuscrito. A los equipos multidisciplinarios de los centros de cáncer públicos del adulto de la Región Metropolitana donde se trataron los pacientes.

\section{Referencias}

1. Autier P, Boniol M, La Vecchia C, Vatten L, Gavin A, Hery C, Heanue M. Disparities in breast cancer mortality trends between 30 European countries: retrospective trend analysis of WHO mortality database. Br Med J 2010; 341: c3620.

2. Edge SB, Byrd DR, Compton CC, Fritz AG, Greene FL, Trotti A, eds.: AJCC Cancer Staging Manual. 7th ed. New York, NY: Springer, 2010, pp 347-76.
3. Protocolos de tratamiento PANDA, Minsal. 2007, 2010.

4. Allemani C, Weir H, Carreira H, Harewood R, Spika $\mathrm{D}$, Wang $\mathrm{X}$, et al and the CONCORD Working Group. Global surveillance of cancer survival 1995-2009: analysis of individual data for 25676887 patients from 279 population-based registries in 67 countries (CONCORD-2). Lancet 2015; 385: 977-1010.

5. Schwartsmann G. Breast cancer in South America: challenges to improve early detection and medical management of a public health problem. J ClinOncol. 2001; 19 (18 Suppl): 118S-124S.

6. Sánchez C, Bustos M, Camus M, Alvarez M, Goñi I, León A, Besa de C P.¿Es curable el cáncer de mama en etapa precoz? Resultados del tratamiento combinado con cirugía, radioterapia y quimioterapia. Rev Med Chil 2007; 135: 427-35.

7. Sánchez C, Acevedo F, Petric M, Galindo H, Domínguez F, León A, et al. Cáncer de mama metastásico. Caracterización de una cohorte según subtipos. Rev Med Chile 2014; 142: 428-35.

8. JansJ, Escudero N, Pulgar D, Acevedo F, Sánchez C, Camus M. Clinicopathologic subtypes and compromise of lymph nodes in patients with breast cancer. Ecancermedicalscience 2014; 8: 448. doi: 10.3332/ecancer.2014.448. eCollection 2014.

9. Shastry M, Yardley DA. Updates in the treatment of basal/triple-negative breast cancer. Curr Opin Obstet Gynecol 2013; 25: 40-8.

10. http://web.minsal.cl/portal/url/item/c4034eddbc96ca6de0400101640159b8.pdf. 